On Skew-Symmetric Splitting and Entropy Conservation Schemes for the Euler Equations

B. Sjogreen, H. C. Yee

October 5, 2009

ENUMATH09

Uppsala, Sweden

June 29, 2009 through July 3, 2009 
This document was prepared as an account of work sponsored by an agency of the United States government. Neither the United States government nor Lawrence Livermore National Security, LLC, nor any of their employees makes any warranty, expressed or implied, or assumes any legal liability or responsibility for the accuracy, completeness, or usefulness of any information, apparatus, product, or process disclosed, or represents that its use would not infringe privately owned rights. Reference herein to any specific commercial product, process, or service by trade name, trademark, manufacturer, or otherwise does not necessarily constitute or imply its endorsement, recommendation, or favoring by the United States government or Lawrence Livermore National Security, LLC. The views and opinions of authors expressed herein do not necessarily state or reflect those of the United States government or Lawrence Livermore National Security, LLC, and shall not be used for advertising or product endorsement purposes. 


\title{
On Skew-Symmetric Splitting and Entropy Conservation Schemes for the Euler Equations
}

\author{
Björn Sjögreen and H. C. Yee
}

\begin{abstract}
The Tadmor type of entropy conservation formulation for the Euler equations and various skewsymmetric splittings of the inviscid flux derivatives are discussed. Numerical stability of high order central and Padé type (centered compact) spatial discretization is enhanced through the application of these formulations. Numerical test on a 2-D vortex convection problem indicates that the stability and accuracy of these formulations using the same high order central spatial discretization are similar for vortex travel up to a few periods. For two to three times longer time integrations, their corresponding stability and accuracy behaviors are very different. The goal of this work is to improve treatment of nonlinear instabilities and to minimize the use of numerical dissipation in numerical simulations of shock-free compressible turbulence and turbulence with shocks
\end{abstract}

\section{Introduction}

Many high resolution numerical schemes for the simulation of turbulence with shocks consist of employing primarily a high order accurate central or Padé (centered compact) spatial discretization in the entire computational domain, and activating a shock-capturing scheme through a flow sensor only in the neighborhood of shocks and in the regions of spurious high frequency oscillations. One example is the filter schemes developed in $[16,17,9,8,14]$. The objective of this paper is to investigate the stability and accuracy behavior of high order spatial central schemes in conjunction with the use of the various skew-symmetric splittings of the inviscid flux derivative or the Tadmor type of entropy conservation formulation for the Euler equations. The flow solutions studied here will therefore be assumed to have a smooth solution.

Due to nonlinear instabilities, solving highly coupled nonlinear conservation laws by spatial centered difference or Padé approximations does not usually lead to a stable method, even when the solution is smooth. Ways to stabilize such methods are to add high order numerical dissipation, or to employ a high pass filter to the solution after each time step. However, in long time integrations and compressible turbulent simulations even small amounts of numerical dissipation can be amplified over time, leading to, e.g., smearing of turbulence fluctuations to un-recognizable forms. An approach to minimize the use of numerical dissipation is to apply these schemes to the split form of the flux derivatives to improve nonlinear stability of the simulation. To understand how this works, consider the the scalar Burgers' equation, $u_{t}+f_{x}=0$ with $f=u^{2} / 2$. The flux derivative can be split into the equivalent form $f_{x}=\frac{1}{2} f_{x}+\frac{1}{2} \frac{\partial f}{\partial u} \frac{\partial u}{\partial x}$. For simplicity of discussion, we discretize the split form by a second-order central scheme

Björn Sjögreen

Lawrence Livermore National Laboratory, Livermore CA94550, e-mail: sjogreen2@1ln1.gov

H. C. Yee

NASA Ames Research Center, Moffett Field, CA94035 e-mail: Helen.M.Yee@nasa.gov 


$$
\frac{d}{d t} u_{j}+\frac{1}{2} u_{j} D_{0} u_{j}+\frac{1}{4} D_{0} u_{j}^{2}=0
$$

The grid is uniform, $x_{j}=(j-1) \Delta x$, with grid spacing $\Delta x$, and $u_{j}(t)$ is an approximation of the solution $u(x, t)$, at the grid point $x_{j}$. The centered difference operator is $D_{0} u_{j}=\left(u_{j+1}-u_{j-1}\right) / 2 \Delta x$. Linearization of (1) around a smooth and bounded solution $\hat{u}_{j}(t)$ leads to the equation

$$
\frac{d}{d t} e_{j}+\frac{1}{2} e_{j} D_{0} \hat{u}_{j}+\frac{1}{2} \hat{u}_{j} D_{0} e_{j}+\frac{1}{2} D_{0} \hat{u}_{j} e_{j}=0
$$

for the small perturbation $e_{j}$. In the scalar product $(u, v)_{h}=\Delta x \sum_{j} u_{j} v_{j}$ and norm $\|u\|_{h}^{2}=(u, u)_{h}$, we obtain

$$
\frac{1}{2} \frac{d}{d t}\|e(t)\|_{h}^{2}=\left(e, e_{t}\right)_{h}=-\frac{1}{2}\left(e, e D_{0} \hat{u}\right)_{h}-\frac{1}{2}\left(e, \hat{u} D_{0} e\right)_{h}-\frac{1}{2}\left(e, D_{0} \hat{u} e\right)_{h} .
$$

The summation by parts property $\left(u, D_{0} v\right)_{h}=-\left(D_{0} u, v\right)_{h}$ eliminates the last two terms to give

$$
\frac{1}{2} \frac{d}{d t}\|e(t)\|_{h}^{2}=-\frac{1}{2}\left(e, e D_{0} \hat{u}\right) \leq C(e, e)_{h}
$$

for a constant $C$ that depends on the maximum spatial derivative of $\hat{u}$. Gronwall's lemma gives the standard well-posedness estimate,

$$
\|e\| \leq K_{1} e^{K_{2} t}
$$

for constants $K_{1}$ and $K_{2}$. Consequently, the linearization of (1) is $L^{2}$ stable. Strang's theorem, see [7], states that if the solution is smooth, and if the method is $p$ th order accurate and smooth, and has an $L^{2}$ stable linearization, then the numerical solution converges with $p$ th order convergence rate. We have thus proved that the split method (1) is convergent as long as no shocks form.

However, because the convergence is up to a fixed time as the grid is refined, it does not necessarily imply that the method is suitable for long time integration. Furthermore, other splittings are possible with different weights on the conservative and non-conservative terms in (1) that do not directly lead to a well-posedness estimate, but turned out to work equally well in numerical experiments. In the absence of better mathematical tools, numerical investigations to assess various possible schemes will be necessary.

In our previous work, non-conservative entropy splitting [15] turned out to be stable and accurate, but when mixed with shock capturing schemes, non-conservative effects sometimes make shocks move with incorrect speeds. As conservative alternatives to entropy splitting, we will consider here the skew-symmetric splitting of Ducros et al. [2,3] and the entropy conservative formulation of Tadmor [11, 12]. Section 2 describes these methods for the Euler equations of compressible gas dynamics. Section 3 reports results from numerical experiments comparing the entropy split scheme, the skew-split scheme, and the entropy conserving scheme. The discussion concentrates of high order central schemes. Padé type of spatial discretizations will not be discussed due to lack of space.

\section{Non-dissipative schemes}

For ease of presentation we will describe non-dissipative schemes applied to the compressible Euler equations in one space dimension. The generalization to three space dimensions is straightforward. The Euler equations are

$$
\mathbf{u}_{t}+\mathbf{f}(\mathbf{u})_{x}=\mathbf{0}
$$

where $\mathbf{u}=(\rho, \rho u, e)$ and $\mathbf{f}(\mathbf{u})=\left(\rho u, \rho u^{2}+p, u(e+p)\right)$. The dependent variables are density $\rho$, momentum $\rho u$, and total energy $e$. The pressure is $p=(\gamma-1)\left(e-\frac{1}{2} \rho u^{2}\right)$, where $\gamma$ is a given constant. The computational domain is $0<x<L$, with periodic boundary conditions at $x=0$ and $x=L$. $\mathbf{u}$ is assumed to be given at the initial time. The grid points $x_{j}=(j-1) \Delta x, j=1, \ldots, N$, where $\Delta x=L /(N-1)$, discretizes the 
computational domain. Undivided difference operators are denoted $\Delta_{+} u_{j}=u_{j+1}-u_{j}, \Delta_{0} u_{j}=\left(u_{j+1}-\right.$ $\left.u_{j-1}\right) / 2$, and $\Delta_{-} u_{j}=\left(u_{j}-u_{j-1}\right)$

\subsection{Skew-symmetric splitting}

Splitting of the derivative of a product in conservative and non-conservative part is done by application of the formula

$$
(a b)_{x}=\frac{1}{2}(a b)_{x}+\frac{1}{2} a b_{x}+\frac{1}{2} a_{x} b,
$$

before discretization. An interesting property is that the split approximation can be written on conservative form,

$$
\frac{1}{2} D_{0}(a b)_{j}+\frac{1}{2} a_{j} D_{0} b_{j}+\frac{1}{2} b_{j} D_{0} a_{j}=\frac{1}{4} D_{+}\left(a_{j}+a_{j-1}\right)\left(b_{j}+b_{j-1}\right),
$$

where $D_{+} u_{j}=\left(u_{j+1}-u_{j}\right) / \Delta x$. (4) can be generalized to arbitrary orders of accuracy if the second order operator $D_{0}$ is replaced by the $2 p$ th order accurate

$$
D_{0 p} u_{j}=\sum_{k=1}^{p} \alpha_{k}^{(p)} D_{0}(k) u_{j} .
$$

The expanded operators are defined as

$$
D_{0}(k) u_{j}=\left(u_{j+k}-u_{j-k}\right) /(2 k \Delta x)
$$

and the coefficients satisfy

$$
\sum_{k=1}^{p} \alpha_{k}^{(p)}=1 \quad \sum_{k=1}^{p} \alpha_{k}^{(p)} k^{2 n}=0, n=1, \ldots, p-1 .
$$

For details see Ducros et al. [2]. Their key idea is to generalize a splitting that leads to kinetic energy conservation for the incompressible flow equations, to compressible flows.

There are many different ways that (3) can be used for the Euler equations. Different splittings are obtained from different ways to write the fluxes as products of two factors, and it is possible to apply splitting to only some of the equations. In the numerical investigations reported in [5], one of the best performing splittings for (2) was (here displayed with second order accuracy)

$$
\begin{aligned}
\frac{d}{d t} \rho_{j}+\frac{1}{2} D_{0} \rho_{j} u_{j}+\frac{1}{2} \rho_{j} D_{0} u_{j}+\frac{1}{2} u_{j} D_{0} \rho_{j} & =0 \\
\frac{d}{d t}(\rho u)_{j}+\frac{1}{2} D_{0} \rho_{j} u_{j}^{2}+\frac{1}{2} \rho_{j} u_{j} D_{0} u_{j}+\frac{1}{2} u_{j} D_{0} \rho_{j} u_{j}+D_{0} p_{j} & =0 \\
\frac{d}{d t} e_{j}+\frac{1}{2} D_{0} u_{j}\left(e_{j}+p_{j}\right)+\frac{1}{2} u_{j} D_{0}\left(e_{j}+p_{j}\right)+\frac{1}{2}\left(e_{j}+p_{j}\right) D_{0} u_{j} & =0 .
\end{aligned}
$$

In three space dimensions the recipe for (7) is to apply (4) to each of the two products in the general three dimensional flux $\mathbf{f}=u_{n} \mathbf{u}+p \mathbf{e}$, where $u_{n}$ is the velocity normal to the cell interface, and $\mathbf{e}=\left(0, k_{1}, k_{2}, k_{3}, u_{n}\right)$, with $\left(k_{1}, k_{2}, k_{3}\right)$ being the cell interface normal.

See [4] for a comparison of splitting methods with different formulations of the energy equation. For a heuristic discussion on aliasing errors for split approximations, see [1].

The homogeneity of the Euler fluxes means that $\mathbf{f}(\mathbf{u})=A(\mathbf{u}) \mathbf{u}$, where $A(\mathbf{u})$ is the Jacobian of $\mathbf{f}(\mathbf{u})$. A natural splitting would therefore be 


$$
\frac{d}{d t} \mathbf{u}_{j}+\frac{1}{2} D_{0} \mathbf{f}_{j}+\frac{1}{2} A_{j} D_{0} \mathbf{u}_{j}+\frac{1}{2} D_{0}\left(A_{j}\right) \mathbf{u}_{j}=0
$$

which is of a form that is more suitable for the norm estimate technique described in Sec. 1 for a scalar problem.

\subsection{Entropy conserving schemes}

Entropy conserving schemes were introduced in in the 1980s. See, e.g., [11]. These schemes are in conservation form, and admit a discrete conservation law for the entropy. An entropy, $E(\mathbf{u})$, and an entropy flux $F(\mathbf{u})$ are two functions satisfying

$$
E_{\mathbf{u}}^{T} A(\mathbf{u})=F_{\mathbf{u}}^{T} .
$$

Here, $E_{\mathbf{u}}$ denotes the gradient of $E$ with respect to $\mathbf{u}$. Furthermore, $E(\mathbf{u})$ is assumed to be a convex function. The entropy variables are defined by $\mathbf{v}=E_{\mathbf{u}}(\mathbf{u})$. Multiplying (2) by $\mathbf{v}^{T}$ gives the entropy equation

$$
\mathbf{v}^{T} \mathbf{u}_{t}+\mathbf{v}^{T} A \mathbf{u}_{x}=E(u)_{t}+F_{\mathbf{u}}^{T} u_{x}=E(u)_{t}+F(u)_{x}=0 .
$$

The entropy flux potential, defined by

$$
\psi=\mathbf{v}^{T} \mathbf{f}-F
$$

has the property that $\mathbf{f}=\psi_{\mathbf{v}}$.

The following construction defines a high order entropy conservation scheme.

Theorem 1. The semi-discrete approximation of a system of conservation laws given by

$$
\Delta x \frac{d}{d t} \mathbf{u}_{j}+\sum_{k=1}^{p} \frac{\alpha_{k}^{(p)}}{k}\left(\mathbf{g}_{j+k / 2}^{(k)}-\mathbf{g}_{j-k / 2}^{(k)}\right)=\mathbf{0},
$$

where $\mathbf{g}_{j+k / 2}^{(k)}$ satisfies

$$
\left(\mathbf{v}_{j+k}-\mathbf{v}_{j}\right)^{T} \mathbf{g}_{j+k / 2}^{(k)}=\psi_{j+k}-\psi_{j}
$$

and where the kth flux differences approximate the flux derivative to second order with a truncation error of even powers of $k \Delta x$,

$$
\mathbf{g}_{j+k / 2}^{(k)}-\mathbf{g}_{j-k / 2}^{(k)}=k \Delta x \mathbf{f}_{x}+k^{3} \Delta x^{3} \phi_{1}+k^{5} \Delta x^{5} \phi_{2}+\ldots,
$$

is $2 p$ th order accurate, and admits a discrete entropy equation

$$
\Delta x \frac{d}{d t} E_{j}+\sum_{k=1}^{p} \frac{\alpha_{k}^{(p)}}{k}\left(H_{j+k / 2}^{(k)}-H_{j-k / 2}^{(k)}\right)=0,
$$

where $H_{j+k / 2}^{(k)}=\frac{1}{2}\left(\left(\mathbf{v}_{j+k}+\mathbf{v}_{j}\right)^{T} \mathbf{g}_{j+k / 2}^{(k)}-\left(\psi_{j+k}+\psi_{j}\right)\right)$. Both (9) and (12) can be cast in conservation form, because

$$
a_{j+k / 2}-a_{j-k / 2}=\Delta_{+}\left(\sum_{m=0}^{k-1} a_{j-k / 2+m}\right)
$$

for any arbitrary grid function $a_{j+k / 2}$ that satisfies $a_{j+k / 2-k}=a_{j-k / 2}$.

Proof. Multiply (9) by $\mathbf{v}_{j}^{T}$ to obtain

$$
\Delta x \frac{d}{d t} E\left(\mathbf{u}_{j}\right)_{t}+\sum_{k=1}^{p} \frac{\alpha_{k}^{(p)}}{k}\left(\mathbf{v}_{j}^{T} \mathbf{g}_{j+k / 2}-\mathbf{v}_{j}^{T} \mathbf{g}_{j-k / 2}\right)=0 .
$$


Rewrite each flux difference as

$$
\begin{aligned}
\mathbf{v}_{j}^{T} \mathbf{g}_{j+k / 2}^{(k)}-\mathbf{v}_{j}^{T} \mathbf{g}_{j-k / 2}^{(k)}= & \frac{1}{2}\left(\mathbf{v}_{j+k}+\mathbf{v}_{j}\right)^{T} \mathbf{g}_{j+k / 2}^{(k)}-\frac{1}{2}\left(\mathbf{v}_{j+k}-\mathbf{v}_{j}\right)^{T} \mathbf{g}_{j+k / 2}^{(k)} \\
& -\frac{1}{2}\left(\mathbf{v}_{j}+\mathbf{v}_{j-k}\right)^{T} \mathbf{g}_{j-k / 2}^{(k)}-\frac{1}{2}\left(\mathbf{v}_{j}-\mathbf{v}_{j-k}\right) \mathbf{g}_{j-k / 2}^{(k)}
\end{aligned}
$$

and use (10) to conclude that

$$
\begin{array}{r}
\mathbf{v}_{j}^{T} \mathbf{g}_{j+k / 2}^{(k)}-\mathbf{v}_{j}^{T} \mathbf{g}_{j-k / 2}^{(k)}=\frac{1}{2}\left(\left(\mathbf{v}_{j+k}+\mathbf{v}_{j}\right)^{T} \mathbf{g}_{j+k / 2}^{(k)}-\left(\mathbf{v}_{j}+\mathbf{v}_{j-k}\right)^{T} \mathbf{g}_{j-k / 2}^{(k)}\right. \\
\left.-\left(\psi_{j+k}+\psi_{j}\right)+\left(\psi_{j}+\psi_{j-k}\right)\right) .
\end{array}
$$

It is clear from (13) that the entropy conservation (12) follows.

It remains to prove that the order of accuracy is $2 p$. Assumption (11) gives

$$
\sum_{k=1}^{p} \frac{\alpha_{k}^{(p)}}{k}\left(\mathbf{g}_{j+k / 2}^{(k)}-\mathbf{g}_{j-k / 2}^{(k)}\right)=\sum_{k=1}^{p} \alpha_{k}^{(p)}\left(\Delta x \phi_{1}+\alpha_{k} k^{2} \Delta x^{3} \phi_{3}+\alpha_{k} k^{4} \Delta x^{5} \phi_{5}+\ldots\right) .
$$

(6) gives

$$
\sum_{k=1}^{p} \frac{\alpha_{k}^{(p)}}{k}\left(\mathbf{g}_{j+k / 2}^{(k)}-\mathbf{g}_{j-k / 2}^{(k)}\right)=\Delta x \mathbf{f}_{x}+\mathcal{O}\left(\Delta x^{2 p+1}\right),
$$

showing that the order of accuracy is $2 p$.

This scheme was also described, although not implemented, in [10].

For a scalar conservation law the simple choice $g_{j+k / 2}^{(k)}=\left(\psi_{j+k}-\psi_{j}\right) /\left(v_{j+k}-v_{j}\right)$ satisfies both (10) and (11). For the one dimensional Euler system $[12,13]$ defined entropy conserving fluxes based on integration in phase space. Here, we instead write $\psi$ as a function of the entropy variables and determine functions $\varphi_{i}$ consistent with the gradient of $\psi$ and satisfying

$$
\left(\psi_{j+k}-\psi_{j}\right)=\varphi_{1}\left(\left(v_{1}\right)_{j+k}-\left(v_{1}\right)_{j}\right)+\ldots+\varphi_{3}\left(\left(v_{3}\right)_{j+k}-\left(v_{3}\right)_{j}\right) .
$$

The definition $\mathbf{g}_{j+k / 2}^{(k)}=\left(\varphi_{1}, \varphi_{2}, \varphi_{3}\right)$ determines an entropy conservative method. As an example, consider the entropy $E(u)=\frac{1+\gamma}{1-\gamma}(\rho p)^{\frac{1}{\gamma+1}}$, which has the entropy flux potential (for explicit expressions for the entropy variables, see [15] or [13])

$$
\psi=-\frac{v_{2}}{v_{3}}\left((\gamma-1)\left(v_{1} v_{3}-v_{2}^{2} / 2\right)\right)^{\frac{1}{1-\gamma}} .
$$

Denote $q=(\gamma-1)\left(v_{1} v_{3}-v_{2}^{2} / 2\right)$, and perform the expansion by repeated use of the rule

$$
\Delta a b=\bar{a} \Delta b+\bar{b} \Delta a
$$

where $\Delta a$ denotes $a_{j+k}-a_{j}$ and $\bar{a}$ denotes $\left(a_{j+k}+a_{j}\right) / 2$. The expansion becomes,

$$
\begin{gathered}
\Delta \psi=\left(-\overline{1 / v_{3}} \Delta v_{2}-\overline{v_{2}} \Delta \frac{1}{v_{3}}\right) \overline{q^{\frac{1}{1-\gamma}}}-\frac{\overline{v_{2}}}{v_{3}} \Delta q^{\frac{1}{1-\gamma}} \\
=\frac{1}{\left(v_{3}\right)_{j+k}\left(v_{3}\right)_{j}}\left(-\overline{v_{3}} \Delta v_{2}+\overline{v_{2}} \Delta v_{3}\right) \overline{q^{\frac{1}{1-\gamma}}}-\frac{\overline{v_{2}}}{v_{3}} \Delta q^{\frac{1}{1-\gamma}}
\end{gathered}
$$

with

$$
\Delta q^{\frac{1}{1-\gamma}}=\frac{q_{j+k}^{\frac{1}{1-\gamma}}-q_{j}^{\frac{1}{1-\gamma}}}{q_{j+k}-q_{j}} \Delta q=\frac{q_{j+k}^{\frac{1}{1-\gamma}}-q_{j}^{\frac{1}{1-\gamma}}}{q_{j+k}-q_{j}}(\gamma-1)\left(\overline{v_{3}} \Delta v_{1}+\overline{v_{1}} \Delta v_{3}-\overline{v_{2}} \Delta v_{2}\right) .
$$


Denoting $Q=\frac{q_{j+k}^{\frac{1}{1-\gamma}}-q_{j}^{\frac{1}{1-\gamma}}}{q_{j+k}-q_{j}}(\gamma-1)$, the final expression becomes

$$
\begin{aligned}
\Delta \psi=\overline{-\frac{v_{2}}{v_{3}}} Q \overline{v_{3}} \Delta v_{1}+ & \left(-\frac{\overline{v_{3}}}{\left(v_{3}\right)_{j+k}\left(v_{3}\right)_{j}} \overline{g^{\frac{1}{1-\gamma}}}+\frac{\overline{v_{2}}}{v_{3}} Q \overline{v_{2}}\right) \Delta v_{2} \\
+ & \left(\frac{\overline{v_{2}}}{\left(v_{3}\right)_{j+k}\left(v_{3}\right)_{j}} \overline{g^{\frac{1}{1-\gamma}}}-\frac{\overline{v_{2}}}{v_{3}} Q \overline{v_{1}}\right) \Delta v_{3} .
\end{aligned}
$$

It is possible to obtain the numerical flux function in standard variables by transforming back from entropy variables. For example, the mass flux for the second order method $(k=1)$ becomes

$$
-\frac{\overline{v_{2}}}{v_{3}} Q \overline{v_{3}}=\bar{u} \overline{\rho\left(p \rho^{-\frac{\gamma}{1+\gamma}}\right)} Q .
$$

The difference quotient $Q$ tends to $p \rho^{\frac{\gamma}{\gamma+1}}$ when $\Delta q$ becomes small. Therefore, this flux is consistent. For comparison, the mass flux in (7) is

$$
\bar{u} \bar{\rho} .
$$

Therefore, apart from the factor $Q$, the entropy conservative scheme can be interpreted as a splitting method. By redefining $Q$ as $\overline{p \rho^{\frac{\gamma}{\gamma+1}}}$, the entropy conservative scheme would become a split scheme, but then perfect entropy conservation would no longer be certain.

\section{Numerical experiments}

The isentropic vortex convection problem for the two dimensional Euler equations has initial data

$$
\begin{aligned}
& \rho=\left(1-\frac{(\gamma-1) \hat{\beta}^{2}}{8 \gamma \pi^{2}} e^{1-r^{2}}\right)^{\frac{1}{\gamma-1}} \\
& u=1-\frac{\hat{\beta}\left(y-y_{0}\right)}{2 \pi} e^{\frac{1-r^{2}}{2}} \\
& v=\frac{\hat{\beta}\left(x-x_{0}\right)}{2 \pi} e^{\frac{1-r^{2}}{2}} \\
& p=\rho^{\gamma}
\end{aligned}
$$

where $r^{2}=\left(x-x_{0}\right)^{2}+\left(y-y_{0}\right)^{2},\left(x_{0}, y_{0}\right)$ is the center of the vortex, and $\hat{\beta}$ is the strength of the vortex. The exact solution consists of the initial data translated with velocity one in the $x$-direction. We solve the isentropic vortex convection problem on the computational domain $0 \leq x \leq 18,0 \leq y \leq 18$ with periodic boundaries. The strength and center of the vortex are $\hat{\beta}=5$ and $\left(x_{0}, y_{0}\right)=(9,9)$, respectively. The grid spacing is $\Delta x=\Delta y=0.25$. All computations use eighth order accurate spatial discretizations with fourth order RungeKutta in time. Figure 1 displays a comparison of the norm of the solution error vs. time for five different methods. The final time of the computation is 180 , which corresponds to 10 periods of vortex convection. D08ES (blue line) denotes the non-conservative entropy splitting of Olsson and Oliger [6, 15] with splitting parameter $\beta=2$, D08SS (red line) denotes the Ducros et al. split scheme (7), D08EC (black line) denotes the Tadmor-like entropy conservative scheme implemented as described in Sect. 2, D08 (green line) denotes the pure centered scheme, and D08CS (light blue line) denotes the natural split scheme (8). All schemes have small errors during the first period. The purely centered approximation, D08, breaks down due to the non-linear instability at a very early time. After two periods D08EC has the smallest error. The error grows to become large after three periods for D08EC, and after around five to six periods for the other schemes. This error is completely dispersive, and the solutions are highly oscillatory for all methods. The skew split schemes, D08SS and D08CS, break down with negative pressure at around time 140. This does not necessarily mean that they 
was discretized by eighth order centered difference operators. The viscosity $\mu=0.001$ is far from resolved on the grid, which has $\Delta x=0.25$. The parabolic time step restriction is not activated. Even with this small dissipation, all methods, except D08, are well behaved. There is no accumulation of high frequency errors. The curves in Fig. 2 are indistinguishable. The viscosity is not large enough to prevent the blow-up of the pure centered scheme. However, increasing the viscosity to $\mu=0.01$, which is also unresolved on the grid, gives more or less identical results with all methods (results not plotted), including the pure centered scheme.

In summary, the non-conservative entropy splitting and the Ducros et al. skew-symmetric split formulations perform the best for this particular smooth flow. However, Ducros et al.'s formulation is conservative and it is applicable to problems containing shock waves.

\section{Acknowledgments}

The work of the first author performed under the auspices of the U.S. Department of Energy by Lawrence Livermore National Laboratory under Contract DE-AC52-07NA27344. The financial support from the NASA Fundamental Aeronautics (Hypersonic) program for the second author is gratefully acknowledged.

\section{References}

1. G. A. Blaisdell, E. Spyropoulos and J.H. Qin, The Effect of the Formulation of Nonlinear Terms on Aliasing Errors in Spectral Methods, Appl. Num. Math., 21 (1996) 207-219.

2. F. Ducros, F. Laporte, T. Souleres, V. Guinot, P. Moinat, B. Caruelle, High-order Fluxes for Conservative Skew-Symmetric-like Schemes in Structured Meshes: Application to Compressible Flows, J. Comput. Phys., 16 (2000) 114-139.

3. W. J. Feiereisen, W. C. Reynolds, and J. H. Ferziger, Numerical Simulation of a Compressible Homogeneous, Turbulent Shear Flow, Report No. TF-13 (1981), Thermosciences Division, Department of Mechanical Engineering, Stanford University.

4. A.E. Honein and P. Moin, Higher Entropy Conservation and Numerical Stability of Compressible Turbulence Simulations, J. Comput. Phys., 201 (2004) 531-545.

5. A.E. Honein and P. Moin, Numerical Aspects of Compressible Turbulence Simulations, Report TF 92, Flow Physics and Computation Division, Department of Mechanical Engineering, Stanford University (2005).

6. P. Olsson and J. Oliger, Energy and Maximum Norm Estimates for Nonlinear Conservation Laws, RIACS Technical Report 94.01, (1994).

7. R.D., Richtmyer and K.W. Morton, Difference Methods for Initial-Value Problems, 2nd ed., John Wiley \& Sons (1967).

8. B. Sjögreen and H. C. Yee, Grid Convergence of High Order Methods for Multiscale Complex Unsteady Viscous compressible Flows, J. Comput. Phys., 185 (2003) 1-26.

9. B. Sjögreen and H. C. Yee, Multiresolution Wavelet Based Adaptive Numerical Dissipation Control for Shock-Turbulence Computation, RIACS Technical Report TR01.01, NASA Ames Research Center (Oct 2000); also, J. Scient. Computing, 20 (2004) 211-255.

10. M. Svärd and S. Mishra Shock Capturing Artificial Dissipation for High-Order Finite Difference Schemes, J. Sci. Comput., 39 (2009) 454-484.

11. E. Tadmor, Numerical Viscosity of Entropy Stable Schemes for Systems of Conservation Laws, I, Math. Comp., 49 (1987) 91-103.

12. E. Tadmor, Entropy Stability Theory for Difference Approximations of Nonlinear Conservation Laws and Related Timedependent Problems, Acta Numer., (2004) 451-512.

13. E. Tadmor and W. Zhong, Entropy Stable Approximations of Navier-Stokes Equations with no Artificial Numerical Viscosity, J. Hyperbolic Diff. Eqn., 3 (2006) 529-559.

14. H. C. Yee, N. D. Sandham, and M. J. Djomehri, Low Dissipative High Order Shock-Capturing Methods Using CharacteristicBased Filters, J. Comput. Phys., 150 (1999) 199-238.

15. H.C. Yee, M. Vinokur, M., and M.J. Djomehri, Entropy Splitting and Numerical Dissipation, J. Comput. Phys., 162 (2000) 33-81.

16. H.C. Yee and B. Sjögreen, Efficient Low Dissipative High Order Scheme for Multiscale MHD Flows, II: Minimization of Div(B) Numerical Error, RIACS Technical Report TR03.10, July, 2003, NASA Ames Research Center; also, J. Scient. Computing, (2005) DOI: 10.1007/s10915-005-9004-5.

17. H.C. Yee and B. Sjögreen, Development of Low Dissipative High Order Filter Schemes for Multiscale Navier-Stokes/MHD Systems, J. Comput. Phys., 225 (2007) 910-934. 